\title{
HOMOTOPY APPROXIMATIONS TO THE SPACE OF KNOTS, FEYNMAN DIAGRAMS, AND A CONJECTURE OF SCANNELL AND SINHA
}

\author{
JAMES CONANT
}

\begin{abstract}
Scannell and Sinha considered a spectral sequence to calculate the rational homotopy groups of spaces of long knots in $\mathbb{R}^{n}$, for $n \geq 4$. At the end of the paper they conjecture that when $n$ is odd, the terms on the antidiagonal at the $E^{2}$ stage precisely give the space of primitive Feynman diagrams related to the theory of Vassiliev invariants. In this paper we prove that conjecture. This has the application that the path components of the terms of the Taylor tower for the space of long knots in $\mathbb{R}^{3}$ are in one-toone correspondence with quotients of the module of Feynman diagrams, even though the Taylor tower does not actually converge. This provides strong evidence that the stages of the Taylor tower give rise to universal Vassiliev knot invariants in each degree.
\end{abstract}

\section{INTRODUCTION}

Consider the space of long knots, $\operatorname{Emb}\left(\mathbb{R}, \mathbb{R}^{n}\right)$, which are a fixed line outside of a compact set. According to the calculus of Goodwillie and collaborators, one can define homotopytheoretic approximations to the space of knots:

$$
e v_{k}: \operatorname{Emb}\left(\mathbb{R}, \mathbb{R}^{n}\right) \rightarrow A M_{k} .
$$

The map $e v_{k}$ induces isomorphisms on homology and homotopy to a larger and larger extent as $k$ increases, provided that $n \geq 4$. In the classical case of $n=3$, we still get knot invariants:

$$
\pi_{0}\left(e v_{k}\right): \pi_{0}\left(\operatorname{Emb}\left(\mathbb{R}, \mathbb{R}^{3}\right)\right) \rightarrow \pi_{0}\left(A M_{k}\right) .
$$

We conjectured in [3] that these maps are actually universal Vassiliev invariants of degree $k-1$ over the integers. The calculations in this paper will show that $\pi_{0}\left(A M_{k}\right)$ is a quotient of the space of primitive Feynman diagrams of degree $k$ that appear in the theory of Vassiliev invariants of knots. (It is a quotient because in the spectral sequence calculations, higher differentials might possibly kill off some of the space.)

To establish this conjecture, we analyze Scannell and Sinha's spectral sequence computations in [4]. They consider a spectral sequence which converges to the rational homotopy groups of the space of long knots, constructed via the Taylor approximations $A M_{n}$, or more precisely, via equivalent cosimplicial models. The main result of this paper is that, when $n$ is odd, the terms along the antidiagonal of the $E^{2}$ page are isomorphic to spaces $\mathcal{A}_{k}^{I}$ of primitive Feynman diagrams.

These spaces $\mathcal{A}_{k}^{I}$ are known to rationally classify primitive Vassiliev invariants of degree $k$ up to lower-degree invariants. The bulk of the current paper is devoted to giving an alternate presentation for $\mathcal{A}_{k}^{I}$, which is hopefully of independent interest. The usual presentation is via trivalent graphs attached to a line segment, modulo the STU, IHX, AS and SEP relations. The new presentation is via connected trivalent trees attached to a line segment modulo the so-called STU ${ }^{2}$, IHX and AS relations. The STU ${ }^{2}$ relation is pictured in Figure 1

In the last section we show that the $E^{1}$ terms on the antidiagonal are isomorphic to the space of trees attached to a directed line segment, modulo IHX relations. On the antidiagonal, passing to the $E^{2}$ page involves dividing by the image of the differential, which has the effect of introducing $\mathrm{STU}^{2}$ relations, thus completing the argument. 
Acknowledgments: This paper arose from discussions with Dev Sinha, and forms part of a joint project with him, Ryan Budney and Kevin Scannell. I'd like to thank them for their helpful discussions. I'd also like to thank Ted Stanford for helpful discussions about alternate presentations of the space of primitive Feynman diagrams.

\section{AN OVERVIEW OF SCANNELL AND SINHA'S RESULT}

Let $\mathcal{B}_{n}^{e}$ be the free graded Lie algebra generated by elements $x_{i j}$ of odd degree where $1 \leq i, j \leq n$, subject to the following relations:

$$
\begin{aligned}
& x_{i j}=-x_{j i} \\
& x_{i i}=0 \\
& {\left[x_{i j}, x_{l m}\right]=0 \text { if }\{i, j\} \cap\{l, m\}=\emptyset} \\
& {\left[x_{i j}, x_{j l}\right]=\left[x_{j l}, x_{l i}\right]=\left[x_{l i}, x_{i j}\right]}
\end{aligned}
$$

Let $M_{d, n}$ be the submodule of the degree $d$ summand of $\mathcal{B}_{n}^{e}$ generated by brackets of elements $x_{i n}$ where all $i<n$ appears as an index. This definition can be rephrased to say that $M_{d, n}$ is the submodule of the degree $d$ summand of $\mathcal{B}_{n}^{e}$ generated by brackets of elements $x_{i j}$ where all of the indices $1, \ldots, n$ appear. (This is a byproduct of [4, Algorithm 5.2].)

There is a differential from $M_{d, n} \rightarrow M_{d, n+1}$ given by:

$$
d=\sum_{i=0}^{n+1}(-1)^{i} \partial^{i}
$$

where

$$
\partial^{l}\left(x_{i j}\right)=x_{\sigma^{l}(i) \sigma^{l}(j)} \text { if } i, j \neq l \text { and } \partial^{l}\left(x_{i j}\right)=x_{i \sigma^{l}(j)}+x_{i+1 \sigma^{l}(j)} \text { if } i=l \text {, where } \sigma^{l}(i)=i \text { if } i<l
$$
and equals $i+1$ if $i>l$.

Theorem 2.1 (Scannell and Sinha). Let $k \geq 4$ be even. There is a spectral sequence which converges, over $\mathbb{Q}$, to

$$
\pi_{*}\left(\operatorname{Emb}\left(I, \mathbb{R}^{k} \times I\right)\right)
$$

whose $E^{1}$ term is given by $E_{-n, d(k-1)+1}^{1}=M_{d, n}$ and whose $d^{1}$ is given by the differential d defined above.

In fact, a recent result [1] indicates that the spectral sequences collapses at the $E^{2}$ term. See Volic's survey paper [5].

For the case of classical knots, $k=2$, there is no convergence result, but Sinha and Scannell conjectured that the submodule of classes along the anti-diagonal correspond to primitive Vassiliev knot invariants.

The main theorem of the current paper is the following. We will define $\mathcal{A}_{n}^{I}$ in the next section.

Theorem 2.2. Let $k$ be even. Then

$$
E_{-(n+1),(n+1)}^{2}=M_{n, n+1} / \operatorname{im}(d) \cong \mathcal{A}_{n}^{I} \otimes \mathbb{Q} .
$$

Here is a different perspective on $d$. In calculating $\partial^{l}$ one takes each instance of $l$ and replaces it by either an $l$ or an $l+1$. Thus there are $2^{k}$ terms in $\partial^{l}(c)$ where $k$ is the number of times the index $l$ appears in $c$.

Proposition 2.3. The differential $d$ is equal to $\sum_{i=1}^{n}(-1)^{i} \tilde{\partial}^{i}$, where the operator $\tilde{\partial}^{l}(c)$ consists of those terms in $\partial^{l}(c)$ in which all indices appear.

Proof. This is straightforward. 


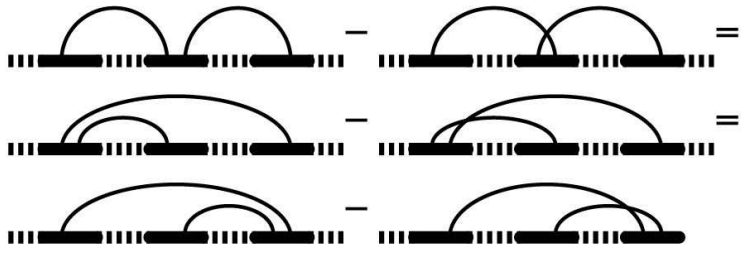

(a)

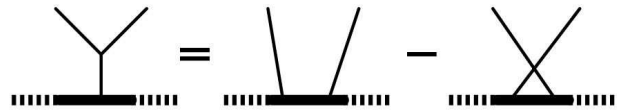

(b)

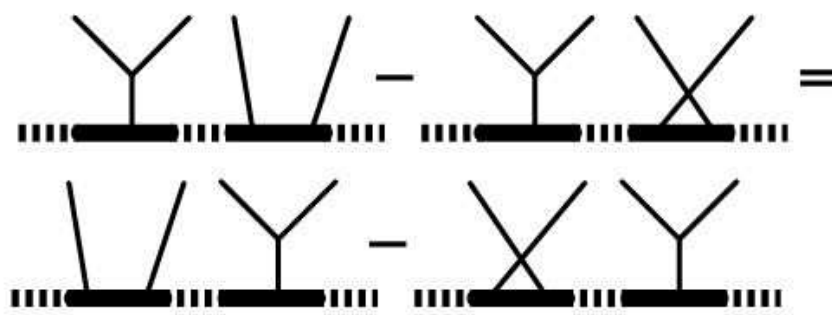

(c)

FIGURE 1. (a) The $4 \mathrm{~T}$ relation. (b) The STU relation. (c) The STU² relation.

\section{OTHER PRESENTATIONS OF $\mathcal{A}_{n}^{I}$}

Here is a quick and dirty review of some spaces of diagrams related to Vassiliev invariants. Let $\mathcal{A}_{n}^{I}$ be the $\mathbb{Z}$-module of chord diagrams. It is generated by diagrams formed by attaching $n$ chords to a directed line segment along distinct pairs of points. The relations are of two forms. The first is called the $4 T$ relation, and is pictured in Figure 1 The second is called the SEP relation and is the relation setting separated diagrams to zero. A separated diagram is one in which there is a vertical line hitting the diagram only at the horizontal line segment, and where there is at least on chord to the right and one chord to the left of this line segment.

Another presentation of this same module is by attaching unitrivalent graphs to a line segment instead of just chords. The total number of vertices, including both the vertices internal to the graphs and the vertices occuring where the trees attach to the line segment, is $2 n$. Also each vertex internal to a tree has a specified cyclic order. The relations are of two forms. The so-called AS relation says that switching the cyclic order at a vertex is the same as multiplication by -1 . The STU relation is as in Figure 1 In this context, elements of $\mathcal{A}_{n}^{I}$ are often called Feynman diagrams (on a directed line segment).

Let's begin by defining some modules closely related to $\mathcal{A}_{n}^{I}$.

\section{Definition 3.1.}

(1) $\tilde{A}_{k, n}^{I}$ is defined to be the module of degree $n$, vertex-oriented, Feynman diagrams on a directed line segment, such that deletion of the line segment yields $k$ tree components.

(2) $\mathcal{A}_{k, n}^{I}$ is the quotient of $\tilde{A}_{k, n}^{I}$ by the relations
(a) IHX
(b) $\mathrm{STU}^{2}$
(c) SEP.

Here IHX is the standard relation which takes place away from the directed line segment. STU ${ }^{2}$ refers to the relation below: one takes an element (called a template) of $\tilde{A}_{k-1, n}^{I}$ or a template which has $k$ components, one of which is a loop, and breaks 
a vertex open via the standard STU relation in two different ways. Pictured is a relation which breaks two vertices apart, but one could also break the same vertex apart using two outgoing edges. Finally SEP sets any separated diagram equal to 0.

Notice that $\mathcal{A}_{n, n}^{I}$ is the usual module of chord diagrams, proven by Bar-Natan to coincide with general Feynman diagrams modulo STU and IHX. So $\mathcal{A}_{n, n}^{I} \cong \mathcal{A}_{n}^{I}$. Note that STU ${ }^{2}$ coincides with the $4 \mathrm{~T}$ relation in this case.

We will use the following fact repeatedly:

Fact 3.2. If $k \leq n-1$, then the $\mathrm{STU}^{2}$ relations are generated by $\mathrm{STU}^{2}$ relations which involve breaking part two distinct vertices of its template.

Proof. In the case $k \leq n-1$ the template has at least 2 trivalent vertices. Any STU ${ }^{2}$ relation involving the same vertex twice can be rewritten as the difference of two $\mathrm{STU}^{2}$ relations, each involving two distinct vertices.

Now we turn to the main theorem of this section:

Theorem 3.3. $\mathcal{A}_{k, n}^{I} \cong \mathcal{A}_{n}^{I}$ for all $1 \leq k \leq n$ except $k=n-1$.

Proof. Here is the strategy of the proof. We will define maps

$$
\Psi_{k}: \mathcal{A}_{k, n}^{I} \rightarrow \mathcal{A}_{k-1, n}^{I} \text { and } \Phi_{k-2}: \mathcal{A}_{k-2, n}^{I} \rightarrow \mathcal{A}_{k-1, n}^{I}
$$

which will make sense for $k \neq n$. The fact that $\Phi_{k-1} \circ \Psi_{k}=$ Id will then imply that each $\Psi_{k}, k>n-1$ is injective. We will also show that each $\Psi_{k}$ is onto. At this point we can then conclude that $\mathcal{A}_{n-2, n}^{I} \cong \mathcal{A}_{n-3, n}^{I} \cong \ldots \cong \mathcal{A}_{1, n}^{I}$. To bridge the last gap, we observe that even though $\Psi_{n}$ is not well-defined, it is well-defined on the module $\tilde{\mathcal{A}}_{n}^{I}$, where no relations are present: $\tilde{\Psi}_{n}: \tilde{\mathcal{A}}_{n, n}^{I} \rightarrow \mathcal{A}_{n-1, n}^{I}$. We then argue that the composition $\Psi_{n-1} \circ \tilde{\Psi}_{n}$ does in fact kill the submodule of relations, giving rise to a well-defined map $\Psi^{\prime}: \mathcal{A}_{n, n}^{I} \rightarrow \mathcal{A}_{n-2, n^{\prime}}^{I}$ which is evidently onto. In a similar vein we define $\Phi^{\prime}=\tilde{\Phi}_{n-2} \circ \Phi_{n-1}$, and argue that $\Phi^{\prime} \circ \Psi^{\prime}=$ Id, implying that $\Psi^{\prime}$ is injective, completing the proof.

The map $\Phi_{k}$ is easier to define. Choose any trivalent vertex which is connected by an edge to the directed line segment, and apply an STU relation to get a difference of two elements in $\mathcal{A}_{k+1, n}^{I}$.

Claim 3.4. $\Phi_{k}$ is well-defined, $k \neq n-2$.

Proof. If one expands a different vertex, then the result is related by an STU ${ }^{2}$-relation. The fact that $\Phi(\mathrm{SEP}) \subset$ SEP is obvious. As for $\Phi\left(\mathrm{STU}^{2}\right)$, we may assume that there are two distinct vertices involved, as pictured in Figure 1. In each of these four terms, calculate $\Phi$ by applying an STU relation to the visible trivalent vertex. The result is zero on the nose. The fact that $\Phi(\mathrm{IHX}) \subset \mathrm{IHX}$ is where we use the hypothesis $k \neq n-2$. If $k=n-1$, there are no IHX relations present, and the result trivially holds. If $k>n-2$, then there are at least three trivalent vertices, and we can split apart one not involved in the IHX relation when calculating $\Phi$, the result obviously lying in the IHX subspace.

Now we define maps $\Psi_{k}: \mathcal{A}_{k, n}^{I} \rightarrow \mathcal{A}_{k-1, n}^{I}, k \neq n$. They are defined in the following way. Given a diagram, $C$, in $\tilde{\mathcal{A}}_{k, n}^{I}$, let $C_{1}$ denote the component whose leg hits the directed line segment first. (Farthest to the left.) $-\Psi(C)$ is a sum of diagrams where each leg of $C_{1}$ is consecutively attached to the legs of the rest of the diagram, moving from right to left until the leg is planted to the left of the rest of the diagram, whereupon the process is repeated for the next leg. See Figure 2. One way to think of the oval notation is that one chooses 


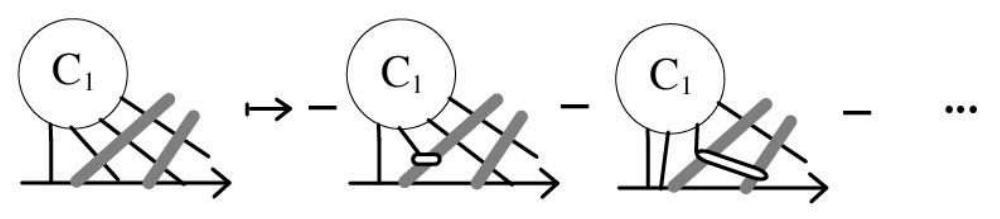

FIGURE 2. The definition of $\Psi$. The circle represents the trivalent tree $C_{1}$ which is attached first to the line segment. The thick grey lines represent regions where legs of other trees may attach. A leg attaching to grey regions by a white oval means a sum of diagrams where the leg attaches to each of the legs inside the grey region.

a horizontal slice of the grey region, transverse to the trees inside of it, and distributes the endpoint of the edge to all of the edges hitting this slice. It is not hard to show that this is well-defined modulo IHX relations. However, an easy way to get a well-definition is to take the horizontal slice to be close to the directed line segment.

Claim 3.5. The following equation holds on the diagrammatic level. That is the domain of $\Psi$ is not divided by any relations, although the range is.

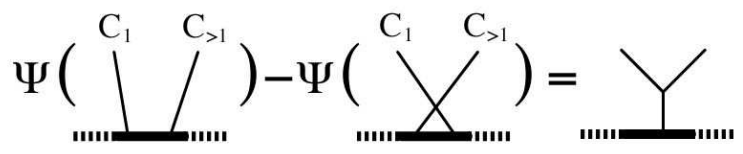

Here the leg labeled $C_{1}$ is part of the tree $C_{1}$, whereas the other leg is part of a different tree.

Proof. Let $L$ be the visible leg of $C_{1}$. Call the legs of $C_{1}$ attached to the left of $L$ prior legs. Applying $\Psi$ to the second term, eventually we get a term formed by crossing the leg $L$ across the leg to its left, creating a term similar to the one on the right, except that all prior legs of $C_{1}$ have been shifted to the left. After this, all the terms from each of the left-hand terms above actually match, and so cancel in pairs. Prior to this point, the terms differ by a transposition of two legs. Using an $\mathrm{STU}^{2}$ relation, turn this transposition into a trivalent vertex in exchange for splitting apart the vertex created by $\Psi$. This gives a sum of terms which are easily seen to combine to give the difference between having the prior legs shifted to the left and having them in their original position.

Claim 3.6. $\Psi$ is well-defined.

Proof. That is, it vanishes on SEP $+\mathrm{IHX}+\mathrm{STU}^{2}$. For SEP, let $R$ be an isolated set of chords not containing $C_{1}$. As the feet of $C_{1}$ drift to the left, attaching to $R$, the isolated property will be destroyed. However, one can verify that the sum of attaching a leg to each of the feet of $R$ is actually a sum of IHX relations. (Similar arguments occur in [2].) Therefore those terms of $\Psi$ in which $R$ is not isolated sum to zero, completing this case.

It is also routine to see that $\Psi(\mathrm{IHX}) \subset \mathrm{IHX}$. Finally we consider $\Psi\left(\mathrm{STU}^{2}\right)$. There are two cases:

Case 1. The $\mathrm{STU}^{2}$ relation does not involve the first component. Without loss of generality, assume our STU ${ }^{2}$ involves two distinct vertices. All of the summands of $\Psi$ which don't involve a leg interacting with a leg in the two active sites, denoted $\alpha$ and $\beta$, of the STU ${ }^{2}$ relation can be grouped to lie in STU ${ }^{2}$. Thus we need only consider dragging a leg across one of the active sites, say $\alpha$. The top of Figure 3 depicts the summands of $\Psi$ where a leg of $C_{1}$ interacts with the legs in the $\alpha$ region. 

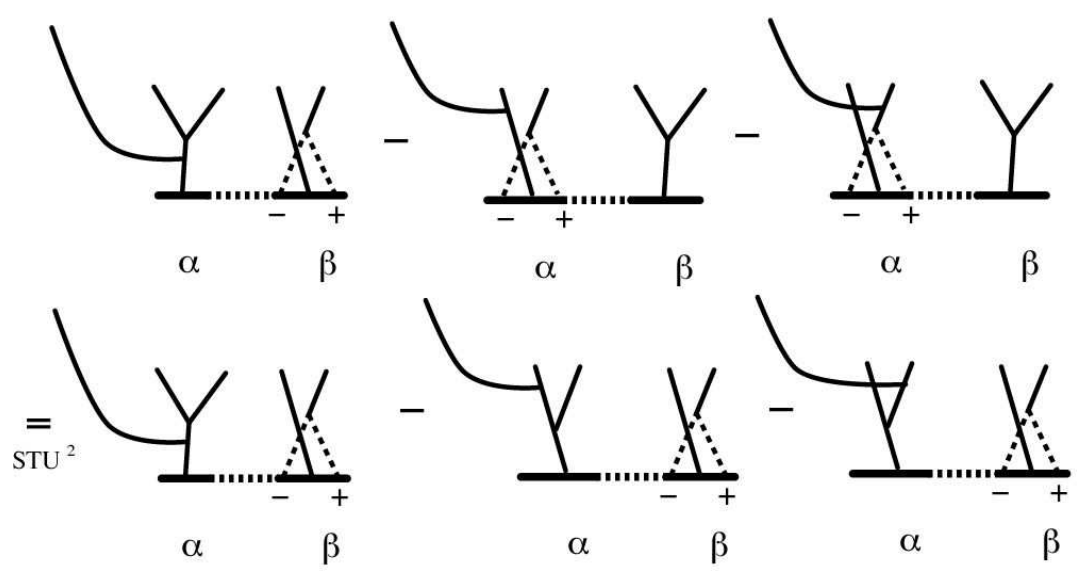

FIGURE 3. From the proof of Case 1. The dotted lines represent a sum of two terms in which the solid line attaches to the bottom line segment in the two indicated ways, with the sign depicted.

Using STU ${ }^{2}$ relations on the last two terms, we get a difference of two IHX relators, which is therefore trivial, as shown in the bottom of Figure 3

Case 2. The $\mathrm{STU}^{2}$ relation does involve the first component. Here there are three subcases.

Subcase 1. The $\mathrm{STU}^{2}$ relation comes from a template graph with a loop being split apart in two ways. Thus all of the feet involved in the $\mathrm{STU}^{2}$ relation come from the component $C_{1}$. Thus one can picture the rest of the graph being slid to the right of $C_{1}$ as opposed to $C_{1}$ being slid to the left, and then apply the argument from Case 1.

Subcase 2. The relation comes from splitting apart two distinct vertices of a template tree, $T$. In this case, the component $C_{1}$ changes. Applying STU to both vertices we get a union of three trees, $T_{1}, T_{2}$ and $T_{3}$. The STU ${ }^{2}$ relation comes in two pairs of terms, where in each pair two of the trees, $T_{i}$, are spliced together and a foot of this resulting tree differs by a transposition with the foot of the third tree in the two terms of the pair. Then Claim 3.5 indicates that $\Psi$ has the effect of converting each pair of terms to the original template tree $T$, with opposite signs, so that the total is zero.

Subcase 3. The relation comes from splitting apart two distinct vertices, each on separate trees, $T_{1}$ and $T_{2}$. Assume that $T_{1}$ has a foot attaching farthest to the left. Using Claim 3.5. $\Psi$ applied to two of the terms in which $T_{1}$ is split apart just gives the template $T$. In the other two terms, we are sliding $T_{1}$ to the left, creating new trivalent vertices along the way, to calculate $\Psi$. Suppose first that the site where $T_{2}$ is being split is to the right of all of the legs of $T_{1}$. Then a similar argument to Claim 3.5 allows us to destroy the created trivalent vertices in exchange for reassembling $T_{2}$. The sum of terms as a result is the difference between attaching $T_{1}$ all the way to the left (which is zero modulo SEP) and attaching $T_{1}$ in the original position (which is the template $T$ ). Now we consider the case when the splitting site for $T_{2}$ is to the left of some legs of $T_{1}$. For the above argument to work, we need to show that the sum of terms where a leg of $T_{1}$ attaches to the two legs in $T_{2}$ 's splitting site is equivalent modulo STU ${ }^{2}$ to the difference of two terms where $T_{2}$ is reassembled, and the leg of $T_{1}$ attaches just before and just after $T_{2}$ 's splitting site, as pictured in Figure 4 . To see that the equality in Figure 4 holds, use STU ${ }^{2}$ relations to split apart the visible trivalent vertices 


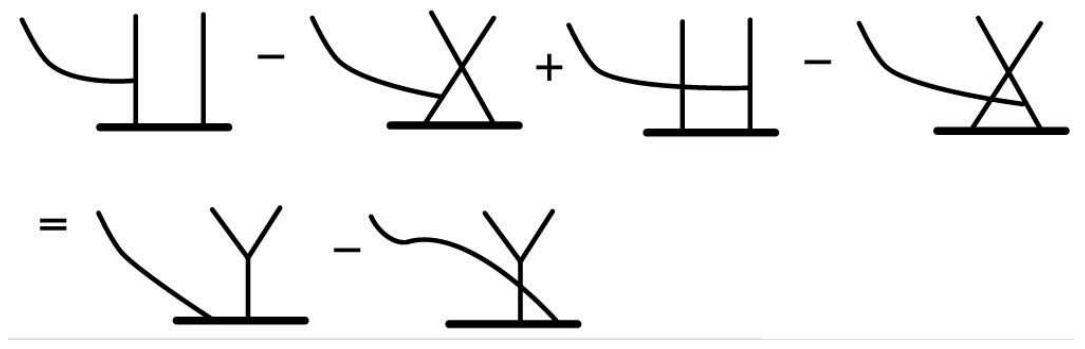

FIGURE 4. From the proof of Subcase 3.

in the picture in exchange for splitting apart some other trivalent vertex, such as the one on $T_{1}$ that we know exists. It is easy to see that equality of the two sides then holds.

Claim 3.7. $\Phi \circ \Psi=\mathrm{Id}$. Hence $\Psi$ is injective.

Proof. This is fairly obvious. $\Psi$ is calculated by dragging feet of $C_{1}$ to the left picking up trivalent vertices as the feet attach to the intervening legs. To calculate $\Phi$ pull apart these very trivalent vertices. The result is the difference between the original diagram and the one gotten by sliding $C_{1}$ all the way to the left, and this latter term lies in SEP.

Let

$$
\Phi^{\prime}: \mathcal{A}_{n-2, n}^{I} \rightarrow \mathcal{A}_{n, n}^{I}
$$

be given by pulling apart two vertices adjacent to the line segment via STU. Let

$$
\Psi^{\prime}: \mathcal{A}_{n, n}^{I} \rightarrow \mathcal{A}_{n-2, n}^{I}
$$

be defined by $\Psi \circ \tilde{\Psi}$ where $\tilde{\Psi}: \tilde{\mathcal{A}}_{n, n}^{I} \rightarrow \mathcal{A}_{n-1, n}^{I}$ is the map $\Psi$ defined on the diagrammatic level. The fact that $\Phi^{\prime}$ is well-defined is straightforward.

Claim 3.8. $\Psi^{\prime}$ is well-defined.

Proof. We need to verify that $4 \mathrm{~T}$ and SEP are in the kernel of $\Psi^{\prime}$. Indeed SEP is already in the kernel of $\tilde{\Psi}$. To show that $4 \mathrm{~T}$ is in the kernel, suppose first that the first chord $C_{1}$ is not involved in the relation. Then all terms except the ones where $C_{1}$ attaches to chords in the relation are clearly in the $4 T \subset S T U^{2}$ subspace. Thus we need to show that the sum of terms where $C_{1}$ attach to the $4 \mathrm{~T}$ chords are in the kernel of $\Psi$. Using Claim 3.5, this is evident. An ad hoc calculation takes care of the case when $C_{1}$ is part of the $4 T$ relation; this calculation is included in the appendix.

Claim 3.9. $\Psi^{\prime}$ is injective.

Proof. This is a consequence of $\Phi^{\prime} \circ \Psi^{\prime}=\mathrm{Id}$, which follows from the following commutative diagrams:

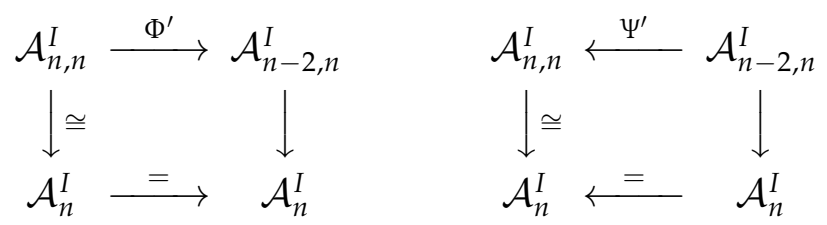

where the vertical arrows are induced by dividing by STU relations. The left-hand isomorphism in each diagram is proven to be so in [2].

Claim 3.10. The maps $\Psi$ and $\Psi^{\prime}$ are surjective. 
Proof. If the first component has a trivalent vertex then Claim 3.5 indicates that it in in the image of $\Psi$. On the other hand, these types of diagrams actually generate $\mathcal{A}_{k, n}^{I}$ for $k<n$, which can be seen as follows. Given a diagram not of this form, it is easy to verify that it is equal to a sum of diagrams, where the right hand foot of the first chord is attached to each of the legs between it and the left-hand foot, and where in each of these terms a trivalent vertex has been split into a sum of two terms.

The fact that $\Psi^{\prime}$ is onto follows since both $\Psi$ and $\tilde{\Psi}$ are onto. (Recall that $\tilde{\Psi}$ has a larger domain than $\Psi$, but that the range is the same.)

\section{Relation to $E^{2}$}

Now let us to return to the question of why $M_{n, n+1} / i m(d) \cong \mathcal{A}_{1, n}^{I} \otimes \mathbb{Q}$. Indeed we will now define a map

$$
\Delta: M_{n, n+1} \rightarrow \mathcal{A}_{1, n}^{I} \otimes \mathbb{Q} .
$$

We can think of $\mathcal{A}_{1, n}^{I} \otimes \mathbb{Q}$ as a vector space spanned by vertex-oriented trivalent trees with leaves that have a specific bijection with $1, \ldots, n+1$, which represent the order in which the leaves attach to the directed line segment. The IHX, AS and STU2 relations can then be interpreted in this context.

We begin with some definitions

Definition 4.1. Given two sets of indices $\alpha$ and $\beta$, define $\alpha \mid \beta$ to be the number of pairs of indices $(a, b)$, of $\alpha$ and $\beta$ respectively where $a>b$.

Definition 4.2. Let $\mathfrak{A}_{n}$ be the vector space spanned by vertex-oriented trivalent trees which have a specified map from the leaves to the set $\{1, \ldots, n+1\}$. That is, we can think of $\mathfrak{A}_{n}$ as spanned by trees with leaves labeled by the numbers $1, \ldots, n+1$ possibly with repetition. We divide by the usual AS and IHX relations.

Define a Lie bracket on $\mathfrak{A}$ by the following rule. Suppose $\alpha$ is the set of numbers labeling a tree $t_{1} \in \mathfrak{A}$, and $\beta$ is the set of number labeling a tree $t_{2} \in \mathfrak{A}$, then $\left[t_{1}, t_{2}\right]$ is defined to be zero unless $\alpha \cap \beta$ consists of a single number, say $i$, in which case it is defined by the equation:

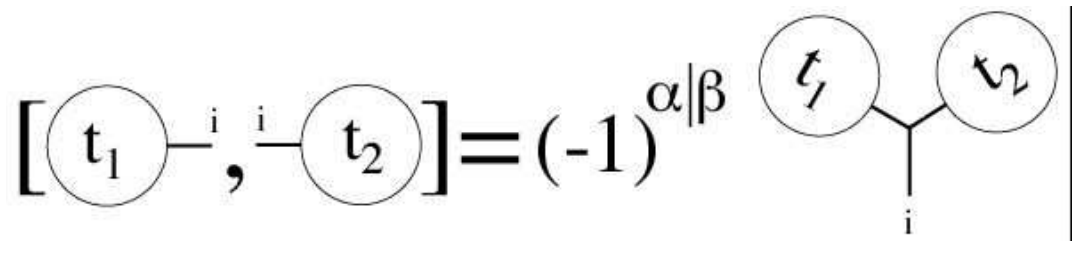

Now we verify that $\mathfrak{A}_{n}$ really is a Lie algebra.

Lemma 4.3. $\mathfrak{A}_{n}$ is a graded Lie algebra, where the grading is given by the number of trivalent vertices minus one.

Proof. We must show that the bracket satisfies the Antisymmetry and Jacobi identities. Let $\alpha_{i}$ be the set of indices involved in $t_{i}$. Antisymmetry is clear, using the identity that $\alpha_{1} \mid \alpha_{2}+$ $\alpha_{2}\left|\alpha_{1}=\right| \alpha_{1}|| \alpha_{2} \mid$.

Here is the Jacobi identity:

$$
\left[t_{1},\left[t_{2}, t_{3}\right]\right]=\left[\left[t_{1}, t_{2}\right], t_{3}\right]+(-1)^{\left|t_{1}\right|\left|t_{2}\right|}\left[t_{2},\left[t_{1}, t_{3}\right]\right]
$$

There are two cases. Either $\alpha_{1}, \alpha_{2}$ and $\alpha_{3}$ meet in a single index or two of them meet in a single index and the third one meets one of the first two in a different index. If neither of 
these two cases hold, all three two-fold brackets are zero, and so the equation trivially holds. If they meet in a single index, then each of the three terms represents attaching a tree with two trivalent vertices to the three leaves of $t_{1}, t_{2}, t_{3}$ labeled with the common index $i$, and labeling the left-over leaf $i$. This exactly corresponds to the Jacobi identity with the possible

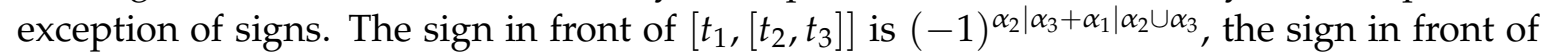
$\left[\left[t_{1}, t_{2}\right], t_{3}\right]$ is $(-1)^{\alpha_{1}\left|\alpha_{2}+\alpha_{1} \cup \alpha_{2}\right| \alpha_{3}}$. Finally the sign in front of $\left[t_{2},\left[t_{1}, t_{3}\right]\right]$ is $(-1)^{\alpha_{1}\left|\alpha_{3}+\alpha_{1} \cup \alpha_{3}\right| \alpha_{2}}$.

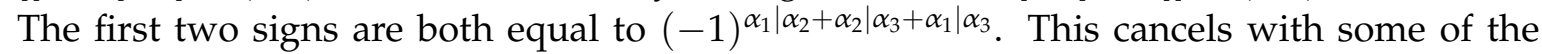
third sign, the residue of which is $(-1)^{\alpha_{3}\left|\alpha_{2}+\alpha_{2}\right| \alpha_{3}}=(-1)^{\left|\alpha_{1}\right|\left|\alpha_{2}\right|}$. This gives the correct signs for the standard Jacobi identity.

The second case is when $\alpha_{1}, \alpha_{2}$ and $\alpha_{3}$ do not meet in a point. For specificity assume that $\alpha_{1}$ and $\alpha_{2}$ meet in the index $i$ and $\alpha_{2}$ and $\alpha_{3}$ meet in the index $j$. Then the term $\left[t_{2},\left[t_{1}, t_{3}\right]\right]$ is zero. The other two terms involve splicing $t_{2}$ to $t_{1}$ and $t_{3}$, and so are equal, and even have the same sign, as we argued above.

Definition 4.4. Let $\overline{\mathcal{A}}_{1, n}^{I}$ be defined like $\mathcal{A}_{1, n}^{I}$ except that we don't divide by $\mathrm{STU}^{2}$ relations. Notice that $\overline{\mathcal{A}}_{1, n}^{I} \otimes \mathbb{Q} \subset \mathfrak{A}_{n}$ as the top degree term.

Definition 4.5. We define a map of Lie algebras $\Delta: \mathcal{B}_{n}^{e} \rightarrow \mathfrak{A}_{n}$ which is given on generators by letting $\Delta\left(x_{i j}\right)$ be the line segment with one end numbered $i$ and the other $j$ with the sign $(-1)^{\{i\} \mid\{j\}}$, unless $i=j$ when we define $\Delta\left(x_{i i}\right)=0$.

Before we show this is well-defined, we give an example.

Example:

$$
\begin{aligned}
\Delta\left(\left[\left[x_{42}, x_{23}\right], x_{13}\right]\right) & =-[[4-2,2-3], 1-3] \\
& =\left[{ }^{4} \mathrm{Y}^{3}, 1-3\right] \\
& ={ }_{2}^{4} \mathrm{H}_{3}^{1} \\
& =\stackrel{ }{\longrightarrow}
\end{aligned}
$$

Proposition 4.6. $\Delta$ is well-defined.

Proof. We must show that $\Delta$ respects the relations from the beginning of Section 2 Clearly $\Delta\left(x_{i j}\right)=-\Delta\left(x_{j i}\right)$, since $(-1)^{\{i\} \mid\{j\}}=-(-1)^{\{j\} \mid\{i\}}$. Also $\Delta\left(x_{i i}\right)=0$ by definition. Relation 3 gets sent to zero because $[i-j, l-m] \in \mathfrak{A}$ is zero if there are no common indices. The trees in relation 4 all get mapped to a trivalent tree with one trivalent vertex and three univalent ones (a "Y"), with leaves labeled $i, j, l$. The signs work out correctly also, for consider the brackets $\left[x_{i j}, x_{j l}\right],\left[x_{l i}, x_{i j}\right],\left[x_{j l}, x_{l i}\right]$. The vertex orientation of the resulting $Y^{\prime} \mathrm{s}$ is the same in all three cases since the cyclic order of indices is the same in all cases. Furthermore, the sign

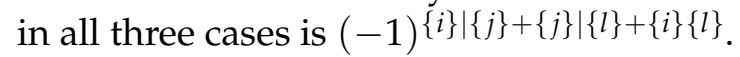

Proposition 4.7. $\Delta$ induces an isomorphism from $M_{n, n+1}$ to $\overline{\mathcal{A}}_{1, n}^{I} \otimes \mathbb{Q}$.

Proof. Let $\nabla: \overline{\mathcal{A}}_{1, n}^{I} \rightarrow M_{n, n+1}$ be defined as follows. Given a tree $t$, labeled by $1, \ldots, n$, think of 1 as the root. For each trivalent vertex, there are two index sets, $\alpha$ and $\beta$ representing the numbers labeling the two branches growing away from the root. For each such vertex consider the sign which is $(-1)^{\alpha \mid \beta}$. Now $\nabla(t)$ is defined to be the iterated commutator formed by replacing each index $k$ by $x_{1 k}$, and interpreting the trivalent vertices of $t$ as brackets, multiplied by the product of signs coming from each vertex.

One must check that $\nabla$ is well-defined, which means that it respects IHX and the antisymmetry relations, which is straightforward. Now $\nabla \circ \Delta=\mathrm{id}_{M_{n, n+1}}$, and $\Delta \circ \nabla=\mathrm{id}_{\overline{\mathcal{A}}_{1, n}^{I}}$. 
Proposition 4.8. The image of $d$ is the subspace of $\mathrm{STU}^{2}$ relations.

Proof. We may take the domain of $d, M_{n, n}$, to be generated by iterated commutators, $c$, in $x_{1 i}$ with a single repeated index $i=k$.

I claim that $M_{n, n}$ is generated by such iterated commutators, with the additional property that $c=\left[c_{1}, c_{2}\right]$ where $c_{1}$ and $c_{2}$ both involve the repeated index $k$. To see this, think of $c$ as a rooted tree in the usual way, and draw a geodesic in the tree connecting the two leaves labeled $x_{1 k}$. We want to rewrite the tree so that the root is distance 1 from this geodesic. Using the Jacobi identity (IHX relation), one can rewrite a tree as a linear combination of two trees where the two new trees have the geodesic one closer to the root. Continue inductively.

Let $c_{1}$ be an iterated commutator in the non-repeated generators $x_{1 k}, x_{1 n_{1}}, \ldots, x_{1 n_{\ell}}$, and let $c_{2}$ be an iterated commutator in the non-repeated generators $x_{1 k}, x_{1 m_{1}}, \ldots, x_{1 m_{s}}$. Let $n_{I}=$ $\left\{n_{1}, \ldots, n_{\ell}\right\}$ and $m_{J}=\left\{m_{1}, \ldots, m_{s}\right\}$, and assume that $n_{I} \cup m_{J} \cup\{1, k\}=\{1, \ldots, n\}$. Then by the preceding paragraph elements of the form $\left[c_{1}, c_{2}\right]$ form a generating set for $M_{n, n}$.

Let $c_{i}[1]$ represent the iterated commutator $c_{i}$ where each $x_{1 j}$ is replaces by $x_{1, j+1}$, and let $c_{i}$ [2] represent the iterated commutator where each $x_{1 j}$ is replaced by $x_{2, j+1}$. Similarly let $c_{i}[k]$ represent the commutator where each $x_{1 j}$ is replaced by $x_{1 j}$ if $j<k$, is replaced by $x_{1, j+1}$ if $j>k$ and by $x_{1 k}$ if $j=k$. Also let $c_{i}[k+1]$ represent the commutator where each $x_{1 j}$ is replaced by $x_{1 j}$ if $j<k$, is replaced by $x_{1, j+1}$ if $j>k$ and by $x_{1, k+1}$ if $j=k$.

Let $c=\left[c_{1}, c_{2}\right]$.Now we claim that

$$
d(c)=-\left[c_{1}[1], c_{2}[2]\right]-\left[c_{1}[2], c_{2}[1]\right]+(-1)^{k}\left[c_{1}[k], c_{2}[k+1]\right]+(-1)^{k}\left[c_{1}[k+1], c_{2}[k]\right]
$$

To see this, note that $d(c)=-\tilde{\partial}^{1}(c)+(-1)^{k} \tilde{\partial}^{k}(c)$, because no other indices $i$ are repeated, implying the operator $\tilde{\partial}^{i}$ is trivial. The first term has the effect of sending all indices $i>1$ to $i+1$ and summing over changing each 1 index to either a 1 or a 2 in all possible ways where both 1 and 2 occur. Similarly the second term converts $k^{\prime}$ s to either $k^{\prime}$ s or $k+1$ 's. This second term is precisely $\left[c_{1}[k], c_{2}[k+1]\right]+\left[c_{1}[k+1], c_{2}[k]\right]$.

Let us calculate the first term. Now consider a term in $\bar{\partial}^{1}(c)$ where some of the 1 's have been converted to 2's. If there are some 1 's that remain in $c_{1}$ and some that remain in $c_{2}$ then $c_{1}$ and $c_{2}$ would have two indices in common, and therefore $c$ would get mapped to zero by $\Delta$. So all of the 1's need to sit in either $c_{1}$ or $c_{2}$ and all of the 2's need to sit in the other one. Thus $\bar{\partial}^{1}(c)=\left[c_{1}[1], c_{2}[2]\right]+\left[c_{1}[2], c_{2}[1]\right]$.

Now we proceed to calculate $\Delta(d(c))$. Let $\Delta\left(c_{1}[1]\right)=t_{1}\left(1, k, n_{1}+1, \ldots, n_{\ell}+1\right)$ where $t_{1}$ represents the underlying tree, and $1, \ldots, k, n_{1}+1, \ldots, n_{\ell}+1$ represent the indices labeling the tree's leaves. Then $\Delta\left(c_{1}[1]\right)=t_{1}\left(2, k+1, n_{1}+1, \ldots, n_{\ell}+1\right)$, which we can abbreviate as $t_{1}\left(2, k+1, n_{I}+1\right)$. To see this, note that the instructions that $\Delta$ gives for gluing the tree together in both cases are the same, and also that even though the index sets differ, they are in the same order, so that the signs which appear will be identical. We can also define $\Delta\left(c_{2}[i]\right)=t_{2}\left(i, k+1, m_{J}+1\right)$ where $i=1,2$. Recalling the definition $\sigma^{k}$ from Section 2. we see that $\Delta\left(c_{1}[i]\right)=t_{1}\left(1, i, \sigma^{k}\left(n_{I}\right)\right)$ where $i=k, k+1$, and $\Delta\left(c_{2}[i]\right)=t_{2}\left(1, i, \sigma^{k}\left(m_{J}\right)\right)$ where $i=k, k+1$. Again, the order types of the index sets are the same, so the signs do not differ.

Now

$$
\begin{aligned}
\Delta(- & {\left.\left[c_{1}[1], c_{2}[2]\right]-\left[c_{1}[2], c_{2}[1]\right]\right)=} \\
& -(-1)^{\{1\} \cup n_{I} \mid\{2\} \cup m_{J}}\left(\left[t_{1}\left(1, k+1, n_{I}\right), t_{2}\left(2, k+1, m_{J}\right)\right]-\left[t_{1}\left(2, k+1, n_{I}\right), t_{2}\left(1, k+1, m_{J}\right)\right]\right)
\end{aligned}
$$


and

$$
\begin{aligned}
\Delta\left((-1)^{k}\left[c_{1}[k], c_{2}[k+1]\right]+(-1)^{k}\left[c_{1}[k+1], c_{2}[k]\right]\right)=(-1)^{k+\{k\} \cup n_{I} \mid\{k+1\} \cup m_{J} \times} \\
\left(\left[t_{1}\left(1, k, \sigma^{k}\left(n_{I}\right)\right), t_{2}\left(1, k+1, \sigma^{k}\left(m_{J}\right)\right)\right]-\left[t_{1}\left(1, k+1, \sigma^{k}\left(n_{I}\right)\right), t_{2}\left(1, k, \sigma^{k}\left(m_{J}\right)\right)\right]\right)
\end{aligned}
$$

In the first two terms we are splicing $t_{1}$ and $t_{2}$ together along the leaves corresponding to the first slot in the parentheses, and the leaves corresponding to the second slot are labeled with $k$ and $k+1$ in both orders. In the second two terms we are splicing the trees together along the leaves corresponding to the second slot, and labeling the first-slot leaves with 1 and 2 in both orders. Thus the sum of the four terms is an STU ${ }^{2}$ relator, up to signs. To see that the signs work out correctly, note the following identities

$$
\begin{aligned}
\{1\} \cup n_{I} \mid\{2\} \cup m_{J} & =\ell+n_{I} \mid m_{J} \\
\{k\} \cup n_{I} \mid\{k+1\} \cup m_{J} & =\{k\}\left|m_{J}+n_{I}\right|\{k\}+n_{I} \mid m_{J} \\
\{k\}\left|n_{I}+n_{I}\right|\{k\} & =\ell \\
\{k\}\left|n_{I}+\{k\}\right| m_{J} & =k-2
\end{aligned}
$$

The first three are fairly straightforward. To see the last identity, note that the left hand side is equal to the number of indices from $n_{I} \cup m_{J}$ which are less than $k$. Since $n_{I} \cup m_{J}$ hits everything except for 1 , we count $k-2$. With these identities, we see that the signs in front of each pair of terms above is opposite, exactly as needed for an STU² relator.

In this way we realize all STU ${ }^{2}$ relations where one of the "splitting sites" is all the way to the left on the line segment. However any STU ${ }^{2}$ relation can be written as a difference of two STU ${ }^{2}$-relations of this type.

The proof of Theorem 2.2 is now complete, since $\Delta$ induces an isomorphism

$$
\Delta: M_{n, n+1} / \operatorname{im}(d) \cong A_{1, n}^{I} \otimes \mathbb{Q} \text {. }
$$

\section{APPENDIX}

In this section we verify that $\Psi^{\prime}: \mathcal{A}_{n, n}^{I} \rightarrow \mathcal{A}_{n-2, n}^{I}$ vanishes on an "initial" $4 T$ relator.

To facilitate our calculations, we need a lemma. Recall the heavy grey line convention from Figure 2

\section{Lemma 5.1.}

(1) The following identity is a consequence of $\mathrm{STU}^{2}$ relations.

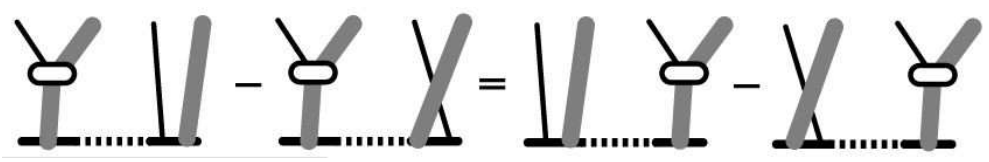

(2) The following identity is a consequence of IHX relations.

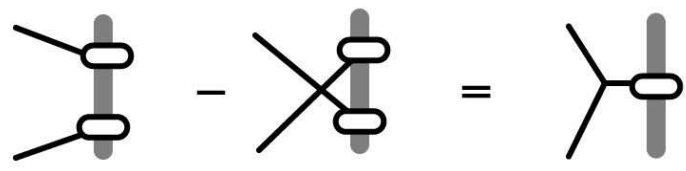

Proof. The first part is straightforward. Each term represents a linear combination of distributing the end of the black edge touching the oval to all of the edges coming through the grey area. Expanding out all four terms above, they naturally group into STU ${ }^{2}$ relations.

The proof of the second part is not much harder, and is encapsulated in Figure 5] The heavy grey line is replaced by the pieces of edges that run through it. 


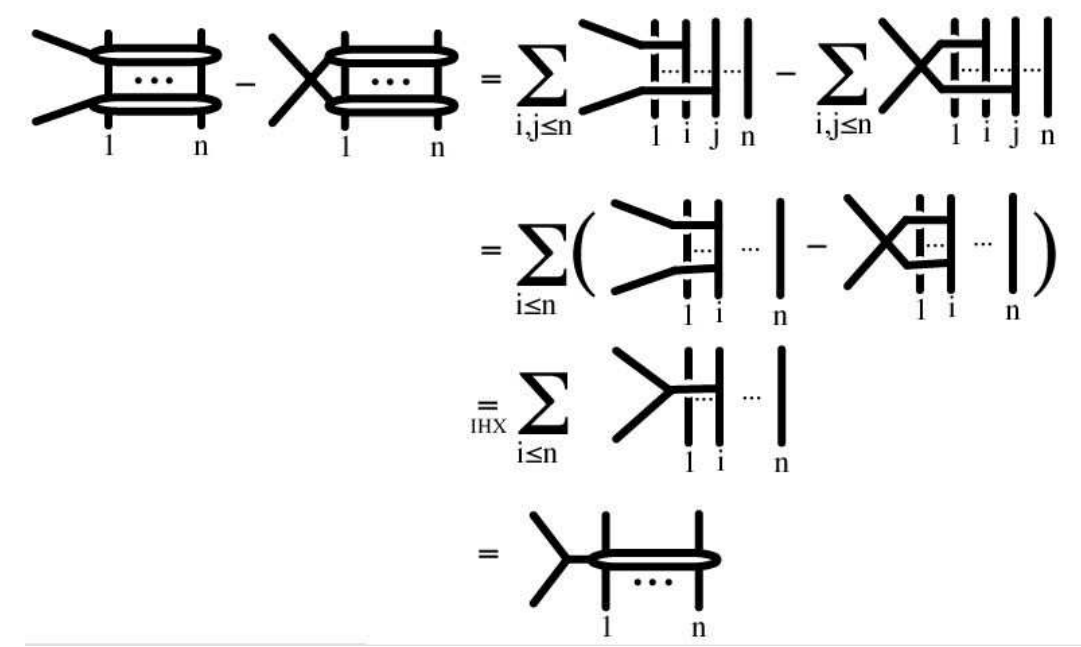

FIGURE 5. The proof of Lemma 5.1(2).

Lemma 5.2. $\Phi^{\prime}$ vanishes on $4 T$ relations involving a chord which attaches farthest to the left.

Proof. The $4 T$ relation is depicted in Figure 1h. Let us first consider a relation of the form coming from the first equality in Figure 1a. At the top of Figure 6 such a $4 T$ relator, involving the left-most chord, is depicted, with heavy grey lines indicating places in the diagram where many edges from a tree may hit, some of which may have multiple endpoints inside the grey region. Then $\tilde{\Psi}$ is applied. The result is reduced modulo STU ${ }^{2}$ relations, and then $\Psi$ is applied again, using Claim 3.5 The result, $\Phi^{\prime}$ of the $4 T$ relator, is the picture at the bottom representing a linear combination of diagrams where the oval depicts summing over puttting the foot on each of the chords inside the grey region. It now remains to show that this linear combination of diagrams is trivial modulo STU ${ }^{2}$ and IHX relations.

The two equations in Figure[7 show that this is true.

Finally, we need to consider a relation from Figure 1a equating the first and third pairs of terms. A straightforward calculation shows that $\tilde{\Psi}$ already sends this to zero. The calculation is simpler, in this case, because the "same" chord is farthest to the left in all four terms. 


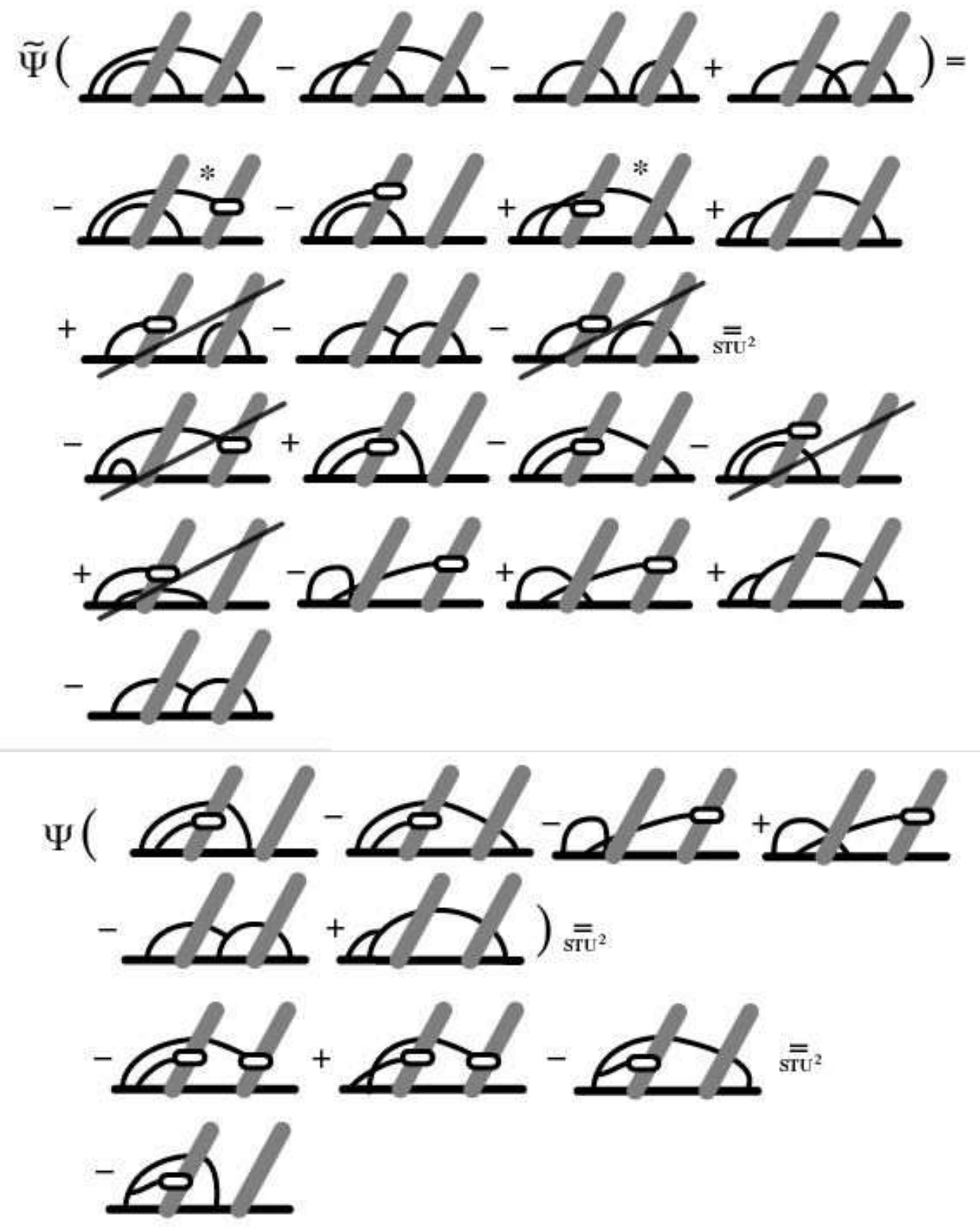

FIGURE 6. Top: Applying $\tilde{\Psi}$ to an "initial" $4 T$ relator. The second equality is an equivalence modulo $\mathrm{STU}^{2}$ relations involving the terms with asterisks. Bottom: Applying $\Psi$ again. The result is $\Phi^{\prime}$ of an initial $4 T$ relator. 


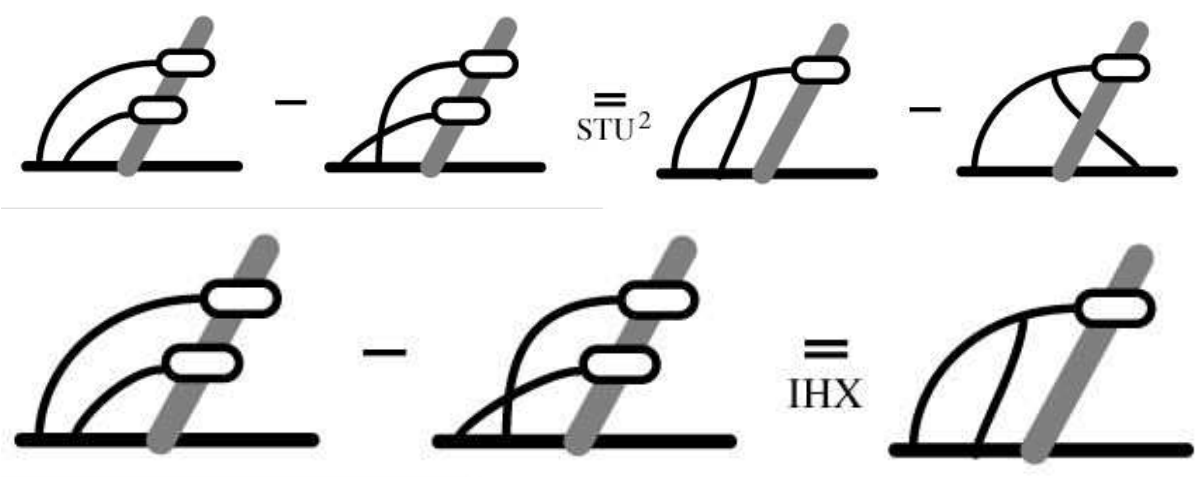

FIGURE 7. From the proof of Lemma[5.2

\section{REFERENCES}

[1] G Arone, P Lambrechts, V Turchin and I Volic, Coformality of the little discs operad and applications to spaces of knots, in preparation.

[2] D Bar-Natan, On the Vassiliev knot invariants Topology 34 (1995), no. 2, $423-472$

[3] R Budney, J Conant, K Scannell and D Sinha, New perspectives in self linking, Adv. Math. 191 (2005), no. 1, 78-113.

[4] K Scannell and D Sinha, A one-dimensional embedding complex, J. Pure Appl. Algebra 170 (2002), no. 1, $93-107$.

[5] I Volic, Calculus of the embedding functor and spaces of knots, math. AT/0601268 